\title{
Tratamiento de la embolia pulmonar masiva con trombectomía reolítica percutánea: resultado intrahospitalario y al seguimiento
}

\author{
Treatment of massive pulmonary embolism with rheolytic percutaneous \\ thrombectomy: inhospital outcome and the follow-up
}

Carlos Fernández Pereira ${ }^{1,2}$, Matías Rodríguez Granillo', Roberto Cristódulo Cortés², Juan Mieres ${ }^{1}$, Paola Renes ${ }^{3}$, Fanny Calvo ${ }^{4}$, Benjamín Ugarte ${ }^{1}$, Jhon Robinson Aldana ${ }^{3}$, Alfredo E. Rodríguez ${ }^{1,2}$

\begin{abstract}
RESUMEN
Objetivo. Evaluar los resultados intrahospitalarios y al seguimiento alejado de pacientes a quienes se realizó embolectomía pulmonar utilizando la trombectomía reolítica con el equipo AngioJet.

Métodos. Este es un registro retrospectivo de pacientes a quienes se realizaron trombectomía pulmonar reolítica desde diciembre de 2009 hasta abril de 2013. Los pacientes fueron tratados por una embolia pulmonar masiva y moderado a severo deterioro de la función ventricular derecha. Los datos registrados incluyen el índice de shock preprocedimiento (frecuencia cardíaca dividida por la presión arterial sistólica) y la presión sistólica de la arteria pulmonar antes y después del procedimiento. Además, el índice de Miller fue calculado con la angiografía pulmonar preprocedimiento y posprocedimiento. Resultados. Catorce pacientes con una media de edad ( \pm DE) de $64,5 \pm 14,3$ años se trataron con embolectomía pulmonar. Todos tenían contraindicación o falla de los trom-

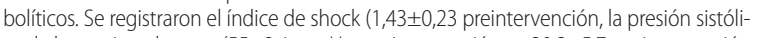
ca de la arteria pulmonar $(55 \pm 8,4 \mathrm{mmHg}$ pre intervención vs. $30,2 \pm 5,7$ posintervención $[\mathrm{p}=0,01]$ ), y el índice de Miller: $20,7 \pm 5$ preintervención vs. $14,2 \pm 4,5$ posintervención $[p=0,01])$.En el seguimiento se realizó ecocardiograma Doppler al mes y los tres meses del procedimiento, y en el contacto fue evaluada la clase funcional de acuerdo a NYHA. A nivel intrahospitalario, un paciente con óbito por sepsis y metástasis múltiples En el

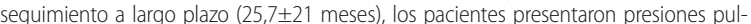
monares similares al posprocedimiento, un óbito por cáncer de próstata, otro en el posoperatorio de bypass femoropoplíteo y otro por ACV isquémico.

Conclusión. El procedimiento de trombectomía pulmonar reolítica fue seguro y efectivo en esta serie de pacientes de alto riesgo. La evolución a largo plazo se relacionó con la patología de base.
\end{abstract}

Palabras claves: embolectomía percutánea reolítica, embolia pulmonar masiva.

\begin{abstract}
Objetives. To evaluate in-hospital and long term outcomes of patients who underwent pulmonary embolectomy using a rheolytic trhombectomy Angio Jet device. Methods. This is a retrospective review of patients who underwent pulmonary rheolitic thrombectomy between December 2009 and april 2013.

Patients were treated having clinical massive $\mathrm{PE}$, and moderate or severe right ventricular dysfunction. Data collected included preprocedure shock index (heart rate divided by systolic blood pressure) and systolic pulmonary artery pressure before and after the procedure. Also Miller score was calculated after pulmonary angiogram.

Results. Fourteen patients with a mean $( \pm$ SD) age of $64.5 \pm 14.3$ years underwent pulmonary embolectomy. All of them were deemed to have contraindications or failed systemic lysis. Patients were measured with shock index (1, 43 $\pm 0,23$ preintervention) and systolic pulmonary artery pressure, the pulmonary pressure improved $(55 \pm 8,4 \mathrm{mmHg}$ preintervention versus $30.2 \pm 5.7$ postintervention $[P=0.01])$,also Miller score improved: $20,7 \pm 5$ preintervention versus $14,2 \pm 4.5$ posintervention $[\mathrm{P}=0.01])$.At one to three months of follow up a new doppler echocardiogram was performed, and then at contact NYHA functional class was assessed. At long term follow-up $(25,7 \pm 21$ months), two patients demonstrated evidence of mild cor pulmonale, in-hospital death was one patient, died due to sepsis, and death at long term follow up was one due to prostate cancer, one after a femoropopliteal by pass and one due to an ischemic stroke.

Conclusion. Percutaneous Pulmonary Rheolytic thrombectomy was safe and effective for the treatment of these high risk patients. The long-term outcome was related to the underlying pathology.
\end{abstract}

Key words: pulmonary rheolytic thrombectomy, massive pulmonary embolism.

Revista Argentina de Cardioangiología Intervencionista 2014;5(2):130-136

\section{INTRODUCCIÓN}

La embolia pulmonar (EP), importante causa de mortalidad con más de 300.000 muertes en el mundo cada año, ${ }^{1}$ todavía nos representa un desafío terapéutico para los médicos, con cuadros clínicos que van desde la enfermedad tromboembólica clínicamente leve hasta la potencialmente mortal embolia pulmonar masiva (EPM). El registro del ICOPER (International Cooperative Pulmonary Embolism Registry) con 2.454 pacientes demostró una tasa de mortalidad del 11\% en la 1ra hora después de la presentación de los síntomas y una mortalidad global del 17,4\% en los 3 meses $^{2}$. Con deterioro mendi CABA, Argentina.

gué. Buenos Aires, Argentina.

3. Cardiología Clínica. Clínica IMA, Adrogué. Buenos Aires, Argentina.

4. Residencia Cardiología Clínica. Sanatorio Otamendi, CABA. Argentina.

$\triangle$ Correspondencia: Carlos Fernández Pereira | cfernandezpereira@centroceci. com.ar

Conflictos de intereses: no existen.

Recibido: 29-12-2013|Aceptado: 13-4-2014. 
TABLA 1.

\begin{tabular}{|c|c|c|c|c|c|c|c|c|c|c|c|}
\hline $\mathrm{p}$ & Edad & Sexo & Comorbilidad & FRTEP & $\mathrm{FC} \times \min$ & \begin{tabular}{|c|} 
PAS \\
$\mathrm{mmHg}$
\end{tabular} & IS & \begin{tabular}{|c|} 
Eco- \\
Doppler
\end{tabular} & TAC & $\mathrm{TLT}$ & Seguimiento \\
\hline 1 & 29 & $\mathrm{~F}$ & Colitis ulcerosa & Embarazo & 150 & 70 & 2,1 & Sí & Sí & Falla, local & 61 \\
\hline 2 & 62 & $F$ & $\begin{array}{l}\text { Enf. coronaria, } \\
\text { HTA, obesidad }\end{array}$ & $\begin{array}{l}\text { Inmovilidad } \\
\text { pos-ATC }\end{array}$ & 100 & 70 & 1,4 & Sí & Sí & Local & 53 \\
\hline 3 & 51 & M & $\begin{array}{l}\text { Ca vejiga, HTA, } \\
\text { obesidad }\end{array}$ & $\begin{array}{l}\text { Ca, posoperat } \\
\text { prostatectomía }\end{array}$ & 103 & 70 & 1,47 & Sí & Sí & Contraindicación & 51 \\
\hline 4 & 64 & $M$ & HTA, DLP & Posoperat RVAo & 115 & 80 & 1,43 & Sí & Sí & Contraindicación & 48 \\
\hline 5 & 85 & $M$ & HTA, obesidad & Edad, inmovilidad & 104 & 100 & 1,04 & Sí & Sí & Contraindicación & 21 \\
\hline 6 & 73 & $\mathrm{~F}$ & HTA, obesidad & Posoperat cadera & 99 & 70 & 1,41 & Sí & Sí & Local & 39 \\
\hline 7 & 63 & M & $\begin{array}{l}\text { HTA, obesidad, } \\
\text { artritis reumatoidea }\end{array}$ & Inmovilidad & 106 & 70 & 1,51 & Sí & Sí & Local & 33 \\
\hline 8 & 64 & M & $\begin{array}{l}\text { Gastristis erosiva, } \\
\text { Ca próstata }\end{array}$ & $\begin{array}{l}\text { Inmovilidad por } \\
\text { viaje aéreo }\end{array}$ & 120 & 80 & 1,5 & No & Sí & Local & 8 \\
\hline 9 & 63 & M & $\begin{array}{l}\text { Ca próstata } \\
\text { metástasis }\end{array}$ & Ca, inmovilidad & 106 & 70 & 1,51 & Sí & Sí & Contraindicación & 1 \\
\hline 10 & 78 & M & HTA, obesidad & \begin{tabular}{|l} 
Inmovilidad, \\
posoperat cole \\
\end{tabular} & 102 & 80 & 1,21 & Sí & Sí & Contraindicación & 2 \\
\hline 11 & 51 & M & $\begin{array}{l}\text { HTA, obesidad } \\
\text { mórbida }\end{array}$ & Inmovilidad & 110 & 70 & 1,57 & Sí & Sí & Contraindicación & 15 \\
\hline 12 & 78 & $\mathrm{~F}$ & HTA, obesidad & $\begin{array}{l}\text { Inmovilidad, } \\
\text { posoperat cadera }\end{array}$ & 108 & 80 & 1,35 & Sí & Sí & Contraindicación & 11 \\
\hline 13 & 54 & M & $\begin{array}{l}\text { HTA, obesidad } \\
\text { mórbida }\end{array}$ & Inmovilidad, EPOC & 100 & 80 & 1,25 & Sí & Sí & Local & 10 \\
\hline 14 & 88 & $F$ & HTA, obesidad, DLP & $\begin{array}{l}\text { Edad, inmovilidad } \\
\text { ATC }\end{array}$ & 100 & 70 & 1,35 & Sí & Sí & Contraindicación & 8 \\
\hline & $64,5 \pm 14,3$ & & & & $108,7 \pm 9,8$ & $75,7 \pm 8,5$ & $1,43 \pm 0,23$ & & & & $\begin{array}{l}25,7 \pm 21 \text { meses } \\
\text { de seguimiento }\end{array}$ \\
\hline
\end{tabular}

FR TEP: factores de riesgo para tromboembolismo pulmonar. Fcxmin: frecuencia cardiaca por minuto. PAS: presión arterial sistólica. IS: índice de Shock. TAC: tomografia axial computarizada. $\mathrm{TLT}$ : trombolítico.

hemodinámico concomitante, la mortalidad hospitalaria se eleva al 31\%, de acuerdo con el estudio de Kasper et al. ${ }^{3}$ Así, en casi dos tercios de los casos, los pacientes inestables morirán dentro de la primera hora, ${ }^{4}$ y de ellos, la EPM demostrada por anatomía patológica representa la mitad de las muertes. ${ }^{5}$

Las modalidades de tratamiento varían ampliamente entre las diferentes categorías de EP, que van desde la anticoagulación sola a la trombólisis sistémica intravenosa (IV), así como la trombectomía mecánica, ya sea percutánea o quirúrgica. ${ }^{6}$.

Los procedimientos de trombectomía mecánica percutánea (PMT) son interesantes como estrategia terapéutica, teniendo en cuenta que hasta el $40 \%$ de los pacientes que se presentan con EP de alto riesgo son demasiado inestables como para someterse a la embolectomía quirúrgica de emergencia o puede presentar contraindicaciones absolutas o relativas a la fibrinólisis.7 Desde diciembre del año 2008 a abril del 2013 realizamos un registro de pacientes con trombectomía reolítica (TR) en pacientes con criterio de embolia pulmonar masiva, presentamos esta serie con los resultados intrahospitalarios y al seguimiento.

\section{MATERIAL Y MÉTODOS}

Registro descriptivo, retrospectivo, desde diciembre de 2008 hasta abril de 2013, donde fueron incluidos 14 pacientes consecutivos con criterios clínicos e imagenológicos de EP masiva, en 2 centros hospitalarios, a través de recolección de datos en fichas diseñadas por el grupo de trabajo. Se realizó trombectomía reolítica utilizándose el dispositivo AngioJet (POSSIS Inc).

El diagnóstico de embolia pulmonar se realizó con base en la presentación clínica y se confirmó mediante angiotomografía computarizada con contraste (los pacientes se sometieron a una tomografía computarizada, si el estado hemodinámico permitía la transferencia a la sala de tomografía computarizada y la administración de medio de contraste endovenoso) o, al menos, un ecocardiograma Doppler transtorácico.

El objetivo de la ecocardiografía era confirmar o brindar elementos altamente sugestivos de EPM, es decir, dilatación y o disfunción del ventrículo derecho. La EPM fue diagnosticada clínicamente en presencia de shock cardiogénico y/o hipotensión sostenida según los criterios de las guías del American College of Chest Physicians, ${ }^{8}$ y de acuerdo con ellos la EPM fue definida como presión arterial menor a $90 \mathrm{mmHg}$ o una caída de la presión arterial sistólica mayor de $40 \mathrm{mmHg}$ por más de 15 min sin otra causa. Además se calculó el índice de shock (IS), que resulta de dividir la frecuencia cardíaca entre la presión arterial sistólica.

La ecocardiografía se realizó de forma sistemática para evaluar el grado de disfunción ventricular derecha. En la sala de Hemodinamia se registraron las presio- 
TABLA 2. Parámetros angiográficos y hemodinámicos.

\begin{tabular}{|r|l|c|c|c|c|c|c|l|l|l|}
\hline $\mathbf{p}$ & Localización & $\mathbf{M}$ pre & $\mathbf{M}$ post & P pre & P post & $\mathbf{S}$ & $\mathbf{M T}$ & TLT local & FVC & Óbito \\
\hline 1 & Tronco-bilat & 32 & 22 & 32 & 17 & sí & no & sí, rTPA & sí & no \\
\hline 2 & Tronco-bilat & 25 & 16 & 51 & 33 & no & no & sí, rTPA & no & no \\
\hline 3 & APD+API & 23 & 15 & 45 & 28 & no & no & no & no & no \\
\hline 4 & APD+API & 21 & 15 & 60 & 32 & no & no & no & no & no \\
\hline 5 & Lobares $d+i$ & 18 & 9 & 58 & 30 & no & no & no & no & sí, 21er mes, ACV isquémico \\
\hline 6 & Lobares $d+i$ & 21 & 12 & 65 & 34 & no & no & sí, sTK & sí & no \\
\hline 7 & APD+API & 16 & 12 & 62 & 28 & no & no & sí, rTPA & no & sí, 8vo mes, cáncer de próstata \\
\hline 8 & APD+API & 23 & 19 & 56 & 35 & no & no & sí, rTPA & no & sí, intrahospitalario, metástasis, sepsis \\
\hline 9 & Tronco-bilat & 26 & 23 & 61 & 42 & no & no & no & no & sí, 2do mes, posoperatorio bypass femoropoplíteo \\
\hline 10 & Lobares $d+i$ & 23 & 15 & 52 & 26 & no & no & no & sí & no \\
\hline 11 & Lobares $d+i$ & 16 & 12 & 54 & 28 & no & no & no & no & no \\
\hline 12 & Lobares $d+i$ & 15 & 9 & 58 & 31 & no & no & no & no & no \\
\hline 13 & Lobares $d+i$ & 16 & 12 & 61 & 34 & no & no & sí, rTPA & no & no \\
\hline 14 & APD+API & 15 & 9 & 55 & 25 & no & no & no & no & no \\
\hline & Media & $20,7 \pm 5$ & $14,2 \pm 4,5$ & $55 \pm 8,4$ & $30,2 \pm 5,7$ & & & & & \\
\hline
\end{tabular}

p: paciente. M pre: Miller preprocedimiento. M post: Miller posprocedimiento. P pre: presión sistólica arteria pulmonar preprocedimiento. P post: presión sistólica arteria pulmonar posprocedimiento. S: sangrado. MT: marcapasos transitorio. TLT local: trombolítico local. FVC: filtro vena cava inferior.

nes pulmonares antes y después del procedimiento. Las angiografías pulmonares se analizaron de acuerdo con el score de Miller (SM) antes (Figura 2) y después del procedimiento.

Brevemente, el SM se calculó con la suma de puntos por índices de obstrucción y déficit de perfusión. Para calcular el índice de obstrucción, se identifican nueve ramas segmentarias en la arteria pulmonar derecha (tres en el lóbulo superior, dos en el medio y cuatro en el inferior) y ocho ramas en la arteria pulmonar izquierda (dos en el lóbulo superior, dos en el medio, y cuatro en el inferior). La presencia de defectos de llenado compatible con trombo adjudica un punto por segmento. Para el índice de perfusión cada pulmón se divide en tres zonas (superior, medio e inferior) y el flujo fue evaluado como sin flujo, 3; severamente reducido, 2; flujo moderadamente reducido, 1; y perfusión nor$\mathrm{mal}, 0$. La suma de las puntuaciones individuales dio el SM, que tiene un máximo de 34, que significa obstrucción vascular completa. El diagnóstico de EPM se confirmó con un SM superior a 10.

Así, el SM fue calculado por la suma de estas puntuaciones que van de cero (la mejor) a 34 . La trombectomía se realizó utilizando un catéter AngioJet ${ }^{\circ}$ (MEDRAD, Inc./Bayer HealthCare, Radiology \& Interventional, Warrendale, PA, EE.UU.), que elimina el trombo por medio del principio de Bernoulli (Figura 1). La TR se realizó por vía venosa femoral percutánea, utilizando un introductor 7 Fr. El catéter de AngioJet se hace avanzar sobre una cuerda coronaria 0,014 en un catéter guía 7 Fr (Figura 3), más comúnmente un catéter Judkins de coronaria derecha (JR4), el cual es guiado hasta el tronco de la pulmonar con un intercambio sobre cuerda de
2,60 m luego de utilizar un catéter de pigtail para realizar la angiografía pulmonar. El catéter guía permite el avance del AngioJet conectado con la unidad de bomba activado durante los pasajes de catéter, en forma lenta, a través del trombo en una dirección desde proximal a distal, luego hacia proximal, bajo control de fluoroscopia. El objetivo del procedimiento era fragmentar y aspirar la mayor cantidad de trombos como sea posible (Figura 4). En seis pacientes, se realizó perfusión local con trombolíticos mediante la función de pulse-spray ubicando trombolíticos en la solución salina de lavado en el equipo, lo cual permite una inyección a gran presión de la mezcla de solución salina más trombolíticos. En la historia clínica se consignaron los factores de riesgo que predisponen la EPM, la terapia médica coadyuvante y las complicaciones intrahospitalarias fueron registradas sistemáticamente.

En el seguimiento a 1-3 meses se realizó ecocardiograma Doppler de control para evaluar función ventricular derecha y presiones pulmonares. En el seguimiento alejado por entrevista o contacto telefónico con el paciente o médico de cabecera se consignaba la clase funcional de acuerdo a la NYHA.

\section{Analisis estadístico}

Las variables continuas se expresan como media $\pm \mathrm{DE}$, y las variables categóricas se representan como número (porcentaje). Dado que las variables continuas no presentaron una distribución normal, se utilizaron pruebas no paramétricas para la comparación. La significación estadística se fijó en el nivel $\mathrm{p}<0,01$. Todos los cálculos se realizaron con SPSS 16.0 (SPSS, Inc., Chicago, IL, EE.UU.) 


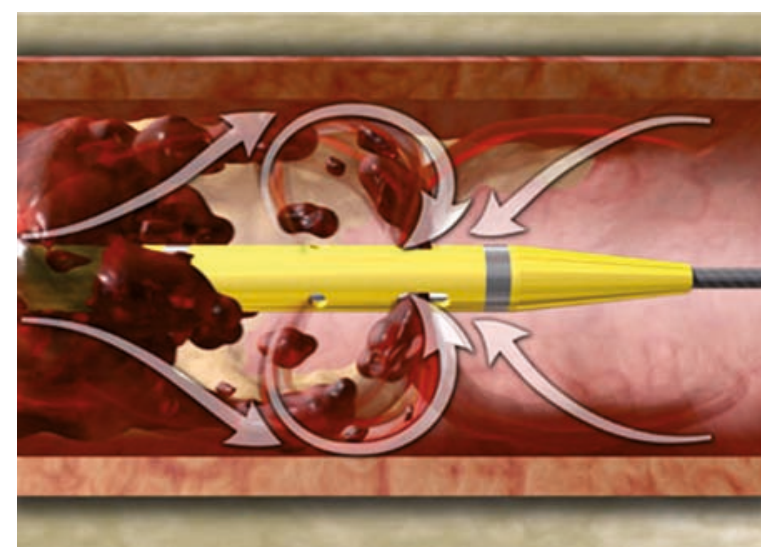

Figura 1.

\section{RESULTADOS}

Catorce pacientes con EPM fueron tratados con embolectomía utilizando el catéter AngioJet; de ellos, cinco en combinación con infusión local de r-tPA (Actilyse $e^{\oplus}$ Boehringer Ingelheim, Ingelheim am Rhein, Alemania) y uno con infusión local de estreptoquinasa (Streptase ${ }^{\circledR}$ ZLB Behring, $\mathrm{GmbH}$, Marburg, Alemania) con la modalidad de pulse-spray.

La edad promedio de los pacientes fue de 64,5 $\pm 14,3$. Nueve fueron hombres (Tabla 1). Solo dos pacientes fueron trasladados de urgencia desde otro centro asistencial para realizar el procedimiento, anticoagulados con heparina. Los factores de riesgo para embolia pulmonar incluyeron obesidad (body mass index mayor de $\left.30 \mathrm{~kg} / \mathrm{m}^{2}\right)(\mathrm{n}=10)$; enfermedad pulmonar obstructiva crónica (EPOC) $(n=1)$; inmovilidad $(n=10)$; enfermedad coronaria con angioplastia previa $(n=2)$; cáncer previo $(n=2)$. Todos los pacientes fueron tratados inicialmente con heparina una vez diagnosticados, a quienes se realizó tomografía computarizada para confirmar el diagnóstico, y en trece de ellos se realizó ecocardiograma Doppler. Cinco pacientes tenían cirugía previa dentro de los 15 días. Ocho pacientes tenían contraindicación absoluta para recibir trombólisis sistémica (cinco con cirugía previa, sangrado gastrointestinal en dos y antecedentes de ACV hemorrágico en uno). En los otros casos se consideraba fallo de la administración de los fibrinolíticos, sin mejoría del cuadro clínico y con mayor descompensación con respecto al ingreso.

Considerando el total de pacientes, hay una mejoría significativa en el índice angiográfico de Miller (Tabla 2): Miller pre $(20,7 \pm 5,02)$, Miller post $(14,3 \pm 4,5)$, $\mathrm{p}<0,001$ (IC95\%: 0,5-7,8), error alfa=0,05.La presión pulmonar sistólica disminuyó luego del procedimiento: PSAP pre $(55 \pm 8,4)$, PSAP post $(30,2 \pm 5,7)$, $\mathrm{p}<0,001$ (IC95\%: 21,4-28,1), error alfa $=0,05$.

El índice de shock previo al procedimiento $(1,43 \pm 0,23)$ confirma el alto riesgo de los pacientes

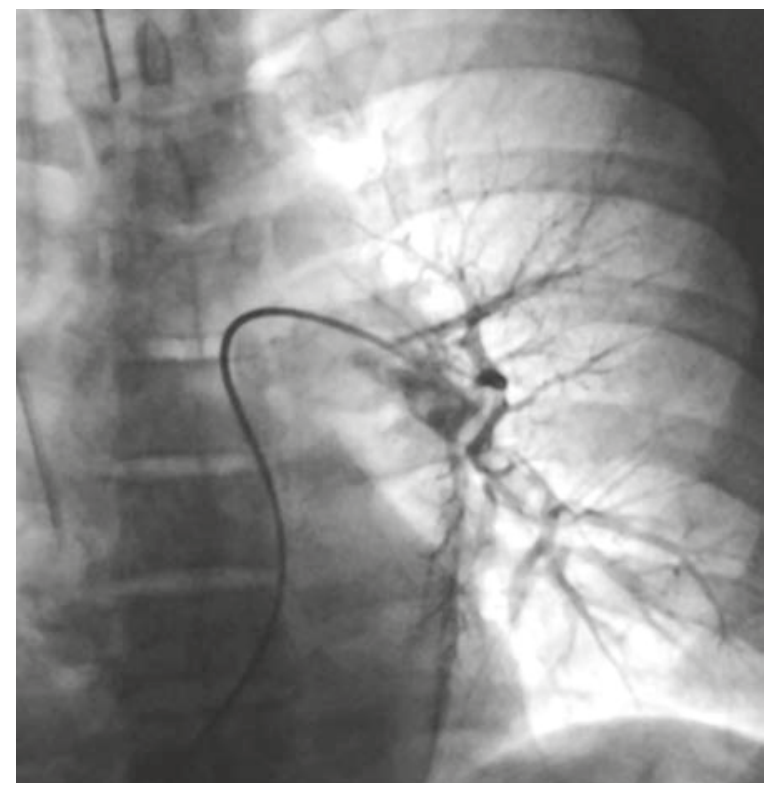

Figura 2.

tratados. No se requirió el uso de marcapasos, y una paciente posparto presentó sangrado luego del uso de $\mathrm{r}-\mathrm{tPa}$ local. Tres pacientes requirieron la colocación de filtro de vena cava.

Al seguimiento alejado de $25,7 \pm 21$ meses, nueve estaban en clase funcional I de la New York Heart Association (NYHA), cuatro en CF II y uno en CF III. Los pacientes en clase funcional II eran pacientes obesos, el paciente en CF III tenía obesidad mórbida. Los ecocardiogramas fueron realizados al mes y tres meses del procedimiento, de acuerdo con el criterio del médico de cabecera, con presiones pulmonares similares al posprocedimiento.

La mortalidad global al seguimiento es del 28,5\%. Un paciente presentó óbito intrahospitalario por sepsis y metástasis de cáncer de próstata, y tres pacientes en el seguimiento: uno a 8 meses secundario a cáncer de próstata, otro a 2 meses del procedimiento en posoperatorio de bypass femoropoplíteo y otro a 21 meses por ACV isquémico.

\section{DISCUSIÓN}

Este registro retrospectivo sugiere que un procedimiento de TR percutánea puede llevarse a cabo en pacientes altamente inestables que se presentan con EP de alto riesgo y shock cardiogénico. De hecho, el procedimiento de TR se realizó en todos los 14 pacientes en ausencia de complicaciones cardiopulmonares relacionadas con el procedimiento. El hallazgo pulmonar principal en la angiografía fue el de las oclusiones de múltiples arterias lobulares, en forma bilateral, responsables de la situación hemodinámica de los pacientes. En la EPM, el tratamiento de elección es la trombólisis IV (bolo e infusión), evidencia clase 1, nivel de evidencia $A .^{10-12}$ 


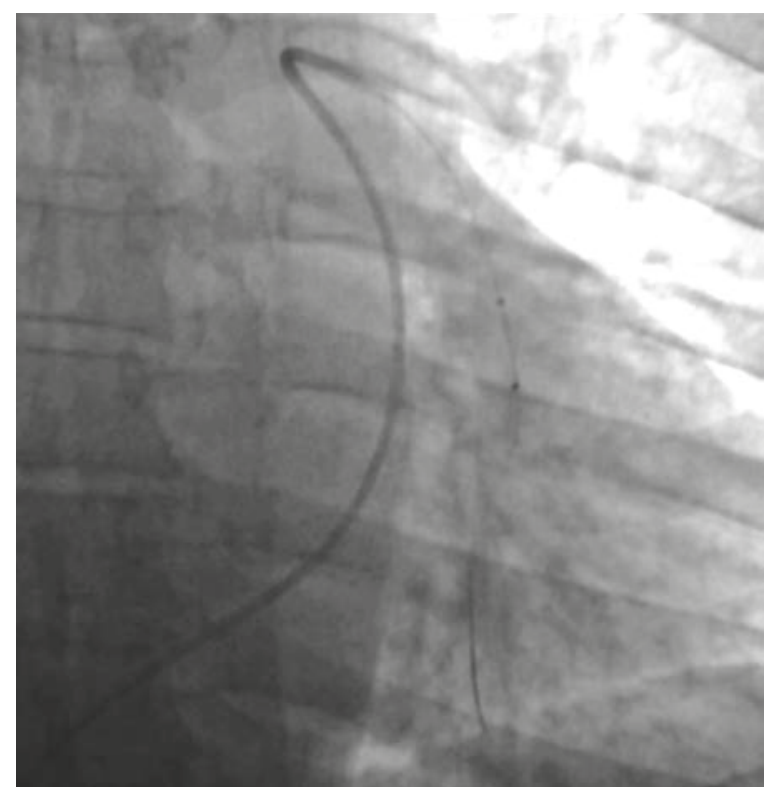

Figura 3.

Sin embargo, los trombolíticos están contraindicados en un grupo importante de pacientes, y aumenta las complicaciones de sangrado $\left(17,6 \%\right.$ en pacientes con EPM) ${ }^{13}$ La embolectomía quirúrgica ${ }^{14}$ es una terapia alternativa para pacientes con EPM en aquellos en los cuales está contraindicada o en el fracaso de los trombolíticos. La mortalidad quirúrgica es extremadamente variable, $y$ en centros especializados con operadores experimentados va desde el 11 al 30\%. La mayoría de los cirujanos rechazan realizar este tratamiento en agudo debido a la alta morbilidad y mortalidad.

Las guías europeas para el manejo de la EPM consideran la trombectomía pulmonar clase $2 \mathrm{~B}$ nivel de evidencia $\mathrm{C}$ como alternativa al tratamiento quirúrgico en pacientes de alto riesgo cuando la trombólisis ha fallado o hay contraindicación. ${ }^{15}$

Estos procedimientos pueden clasificarse en trombectomía por aspiración, trombectomía por fragmentación, y trombectomía reolítica, que se diferencian de los otros procedimientos de trombectomía. ${ }^{16}$

La TR ha probado su eficacia y seguridad a nivel coronario, ${ }^{17,18}$ así como también a nivel extracoronario, periférico arterial y enfermedad tromboembolica venosa. ${ }^{19,20}$ Sin embargo, su uso en casos de EPM de alto riesgo hasta ahora no ha sido evaluado de forma prospectiva. En consecuencia, la evidencia relacionada con el uso del AngioJet consiste únicamente en informes de casos esporádicos y algunos series retrospectivas, ${ }^{21-23}$ incluyendo los primeros 2 casos en procedimientos de urgencia reportados en Argentina ${ }^{24}$.

Zeni et al. en 2003 aplicaron la TR con AngioJet en 17 pacientes que se presentaron con EPM. En esta serie, el procedimiento fue asociado generalmente con la administración trombólisis intrapulmonar, el cual, en última instancia, enmascara cuál sería el efecto aislado del procedimiento $^{25}$

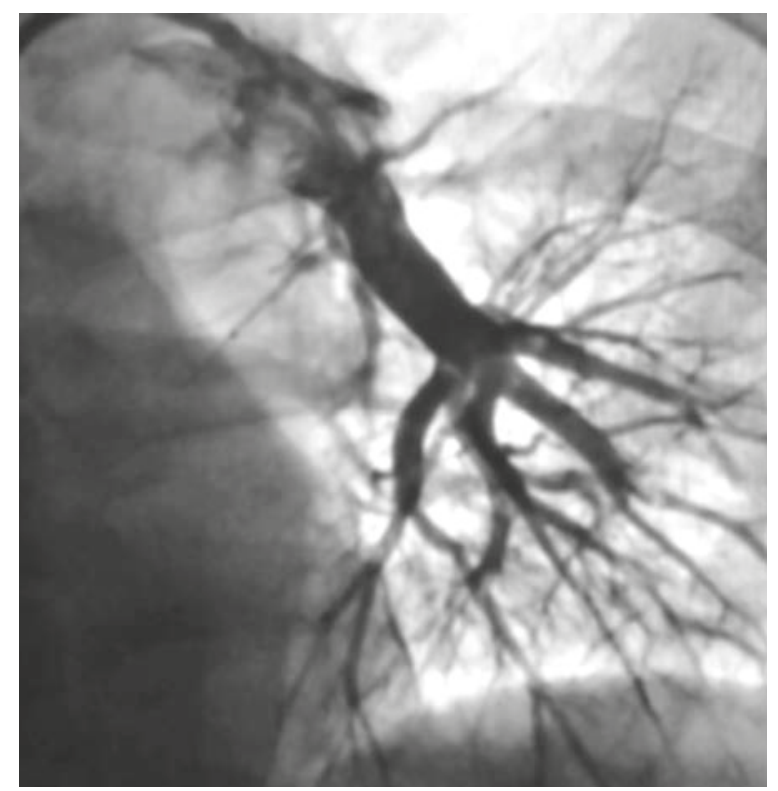

Figura 4.

En forma similar, el estudio retrospectivo de Chechi et al. sugiere seguridad y eficacia de este procedimiento de TR en 51 pacientes con EPM y submasiva. ${ }^{26}$

Mas recientemente, también Nassiri et al. han reportado el uso de la TR en su modo pulse spray en 15 pacientes que se presentaron en su mayoría como EP no catalogados como de alto riesgo ( $93 \%$ de la población tratada). ${ }^{27}$ En el estudio de Chechi et al., dos tercios de esos pacientes incluidos, que es la mayor serie con EPM utilizando la TR, fueron pacientes con presentación clínica de EPM de alto riesgo, que se define como un índice de Miller $\geq 17$ en la angiografía. Así, el índice de Miller medio de 19 que se describe en esa serie, que incluyó $<30 \%$ de los pacientes en shock cardiogénico, era de seis puntos inferior a la calculada para la población del estudio de muy alto riesgo de Bonvini et al, ${ }^{28}$ en el cual 7 de los 10 pacientes presentaron óbito intrahospitalario. En el trabajo de Bonvini et al. se estudió con un IM de 25 como media, no se consideró el score de Miller como parámetro más valioso para definir la EPM de alto riesgo. Se consideraron exclusivamente los parámetros hemodinámicos, como el índice de shock, como criterios de inclusión para el estudio. De hecho, los pacientes con EPM y disfunción ventricular derecha con hipotensión que rápidamente era mejorada con aportes de líquido en forma agresiva sin necesidad de apoyo de catecolaminas no fueron considerados elegibles para ese estudio. Las consecuencias directas de estos criterios de inclusión selectiva fueron el muy lento reclutamiento de pacientes (un paciente cada dos meses) y que los pacientes incluidos estaban en condición muy grave: el 100\% en shock cardiogénico, el $80 \%$ con ventilación mecánica por una dificultad respiratoria grave, y el $60 \%$ ya habiendo experimentado un episodio de paro cardíaco que necesitaba RCP antes del procedimiento de TR. 
La diferencia de gravedad de los pacientes dentro del alto riesgo probablemente explica por qué la diferencia en las tasas de mortalidad en estos estudios (de la serie de Zeni: 11,8\%; serie de Chechi: 15,7\%; serie de Nassiri: 0\%), más bajos que los que hemos observado en el estudio de Bonvini.

En contraste con las arterias coronarias, donde la TR es muy eficaz para los trombos ricos en plaquetas, el trombo en las arterias pulmonares se origina de la circulación venosa, con trombos con abundante fibrina, lo cual sugiere que pueden ser más antiguos (días o semanas), más organizados y más difíciles de fragmentar y aspirar. En esta situación puede ser útil la técnica del pulse spray. ${ }^{29}$

De esta forma, Hubbard, Nassiri y Ferrigno et al. han obtenido una tasa muy alentadora en la mortalidad hospitalaria (9,1\%, $0 \%$ y $6,3 \%$, respectivamente) mediante el uso de esta técnica pulse-spray en caso de EPM y submasiva. El concepto de pulse-spray es el de mezclar con fuerza e inyectar una pequeña cantidad de trombolíticos directamente en el trombo, lo cual puede contribuir a disolverlo. Este método, que fue utilizado en seis pacientes de nuestra serie, merece una evaluación en el futuro con mayor cantidad de pacientes como una posible solución para mejorar los resultados. ${ }^{31,32}$

Finalmente, a pesar de los datos referidos a las ventajas de la TR, existen dudas con respecto a posibles efectos adversos observados en otras series. Una de ellas, la hemólisis producida por la fragmentación del coágulo, con la liberación de substancias neurohormonales como la adenosina y la bradiquinina a nivel de los vasos pulmonares, los cuales podrían producir bradicardia e hipotensión. ${ }^{33}$

\section{BIBLIOGRAFÍA}

1. Tapson VF. Acute pulmonary embolism. NEngl J Med 2008;358:1037-1052.

2. Goldhaber SZ, Visani L, De Rosa M, et al. Acute pulmonary embolism: clinical outcomes in the International Cooperative Pulmonary Embolism Registry (ICOPER). Lancet 1999;353:1386-1389.

3. Kasper W, Konstantinides S, Geibel A, et al. Management strategies and determinants of outcome in acute major pulmonary embolism: results of a multicenter registry. J Am Coll Cardiol 1997;30:1165-1171.

4. Stein PD, Henry JW. Prevalence of acute pulmonary embolism among patients in a general hospital and at autopsy. Chest 1995;108:978-981.

5. Coon WW, Coller FA. Clinicopathologic correlation in thromboembolism. Surg Gynecol Obstet 1959;109:259-269.

6. Kucher N, Goldhaber SZ. Management of massive pulmonary embolism. Circulation 2005; 112:e28-32

7. Jaff MR, MCMurtry MS, Archer SL, Cushman M, et al. Management of massive and submassive pulmonary embolism, iliofemoral deep vein thrombosis, and chronic thromboembolic pulmonary hypertension: a scientific statement from the American Heart Association. Circulation 2011;123:1788-1830.

8. Kearon C, Kahn SR, Agnelli G, et al. Antithrombotic therapy for venous thromboembolic disease: American College of Chest Physicians Evidence-Based Clinical Practice Guidelines (8th Edition). Chest 2008;133(6 Suppl):454S-545S

9. Miller $G A$, Sutton $G C$, Kerr IH, et al. Comparison of streptokinase and heparin in treatment of isolated acute massive pulmonary embolism. $\mathrm{Br}$ Med J 1971;2:681-684.

10. Torbicki A, Perrier A, Konstantinides S, et al. Guidelines on the diagnosis and management of acute pulmonary embolism: the Task Force for the
De modo secundario a la hemólisis estarían relacionados también la hiperkalemia y la hemoglobinuria. ${ }^{34}$ No se observaron esos efectos en nuestros pacientes, por consiguiente no se necesitó marcapasos transitorios ni se observó deterioro de la función renal con respecto al basal.

\section{LIMITACIONES DEL ESTUDIO}

Las principales limitaciones de este estudio son el número de pacientes, los procedimientos realizados mayoritariamente (13/14) en un solo centro, todos derivados por la unidad coronaria por lo cual puede haber un sesgo de inclusión y la administración en seis pacientes de trombolíticos locales en combinación con la tromboaspiración reolítica.

Sin embargo, este registro observacional tiene como principal objetivo establecer la posibilidad técnica de realizar TR en un grupo de pacientes de alto riesgo, así como confirmar la ausencia de complicaciones mayores relacionadas con el dispositivo. Por otra parte, un punto fuerte del mismo es que el procedimiento de TR fue estandarizado y siempre realizado por los mismos operadores experimentados.

\section{CONCLUSIÓN}

La trombectomía reolítica percutánea, en esta serie de pacientes con criterios de trombólisis pero con fracaso o contraindicación, fue un medio seguro y efectivo para el tratamiento de la TEP masiva a nivel intrahospitalario y en el seguimiento inmediato. La evolución a largo plazo fue relacionada a la patología de base.

Diagnosis and Management of Acute Pulmonary Embolism of the European Society of Cardiology (ESC). Eur Heart J 2008;29:2276-2315.

11. Jaff MR, McMurtry MS, Archer SL, et al. Management of massive and submassive pulmonary embolism, iliofemoral deep vein thrombosis, and chronic thromboembolic pulmonary hypertension: a scientific statement from the American Heart Association. Circulation 2011;123:1788-1830.

12. Kearon C, Kahn SR, Agnelli G, et al. Antithrombotic therapy for venous thromboembolic disease: American College of Chest Physicians Evidence-Based Clinical Practice Guidelines (8th Edition). Chest 2008; 133:454S-545S.

13. Kucher N, Rossi E, De RM, Goldhaber SZ. Massive pulmonary embolism Circulation 2006:113:577-582.

14. Stein PD, Alnas M, Beemath A, Patel NR. Outcome of pulmonary embolectomy. Am J Cardiol 2007;99:421-423.

15. Torbicki A, Perrier A, Konstantinides SV, et al. Guidelines on the management and diagnosis of acute pulmonary embolism. Eur Heart $\rfloor$ 2008;29:2276-2315.

16. Kucher N, Windecker S, Banz Y, et al. Percutaneous catheter thrombec tomy device for acute pulmonary embolism: in vitro and in vivo testing. Radiology 2005;236:852-858

17. Migliorini A, Stabile A, Rodríguez AE, et al. Comparison of AngioJet rheolytic thrombectomy before direct infarct artery stenting with direct stenting alone in patients with acute myocardial infarction. The JETSTENT trial. J Am Coll Cardiol 2010;56:1298-1306.

18. Kuntz RE, Baim DS, Cohen DJ, et al. A trial comparing rheolytic thrombec- 
tomy with intracoronary urokinase for coronary and vein graft thrombus (the Vein Graft AngioJet Study [VeGAS 2]). Am J Cardiol 2002;89:326-330.

19. Kasirajan K, Haskal ZJ, Ouriel K. The use of mechanical thrombectomy devices in the management of acute peripheral arterial occlusive disease. J Vasc Interv Radiol 2001;12:405-436.

20. Rao AS, Konig G, Leers SA, et al. Pharmacomechanical thrombectomy for iliofemoral deep vein thrombosis: an alternative in patients with contraindications to thrombolysis. J Vasc Surg 2009;50:1092-1098.

21. Siablis D, Karnabatidis D, Katsanos K, etal. AngioJet rheolyticthrombectomy versus localintrapulmonary thrombolysis in massivepulmonaryembolism: a retrospective data analysis. J Endovasc Ther 2005;12:206-214.

22. Margheri $M$, Vittori $G$, Vecchio $S$, et al. Early and long-term clinical results of AngioJet rheolytic thrombectomy in patients with acute pulmonary embolism. Am J Cardiol 2008;101:252-258.

23. Arzamendi D, Bilodeau L, Ibrahim R, et al. Role of rheolytic thrombectomy in massive pulmonary embolism with contraindication to systemic thrombolytic therapy. Eurolntervention 2010;5:716-721.

24. Fernández Pereira C, Curotto V, Rodríguez Pagani C, Renes MP, Rodríguez AE. Embolectomía percutánea reolítica en la tromboembolia pulmonar masiva. Rev Argent Cardiol 2010;78:442-444.

25. Zeni PT Jr, Blank BG, Peeler DW. Use of rheolytic thrombectomy in treatment of acute massive pulmonary embolism. I Vasc Interv Radiol 2003:14:1511-1515

26. Chechi T, Vecchio S, Spaziani G, et al. Rheolytic thrombectomy in patients with massive and submassive acute pulmonary embolism. Catheter Cardiovasc Interv 2009;73:506-513.

27. Nassiri N, Jain A, McPhee D, et al. Massive and submassive pulmonary embolism: experience with an algorithm for catheter-directed mechanical thrombectomy. Ann Vasc Surg 2012;26:18-24.

28. BonviniR, Roffi M, BounameauxH, etal. AngioJet rheolytic thrombectomy in patients presenting with high-risk pulmonary embolism and cardiogenic shock: a feasibility pilot study. Eurolntervention 2013;8:1419-1427.

29. Hubbard J, Saad WE, Sabri SS, et al. Rheolytic thrombectomy with or without adjunctive indwelling pharmacolysis in patients presenting with acute pulmonaryembolism presenting with right heartstrain and/or pulseless electrical activity. Thrombosis 2011;2011:246410.

30. Ferrigno L, BlochR, ThrelkeldJ, etal.Management ofpulmonaryembolism with rheolytic thrombectomy. Can Respir J 2011;18(4):e52-e58.

31. Schmitz-Rode T, Kilbinger M, Gunther RW. Simulated flow pattern in massive pulmonary embolism: significance for selective intrapulmonary thrombolysis. Cardiovasc Intervent Radiol 1998;21:199-204.

32. Kuo WT. Endovascular therapy for acute pulmonary embolism. J Vasc Interv Radiol 2012;23:167-179. e4; quiz 79.

33. Dwarka D, Schwartz SA, Smyth SH, et al. Bradyarrhythmias during use of the AngioJet system. J Vasc Interv Radiol 2006;17:1693-1695.

34. DukkipatiR, Yang EH, Adler S, et al. Acute kidney injury caused by intravascular hemolysis after mechanical thrombectomy. Nat Clin Pract Nephrol 2009;5:112-116. 